\title{
Chapter 6 \\ On Different Aspects of Network Analysis in Systems Biology
}

\author{
Amphun Chaiboonchoe, Wiktor Jurkowski, Johann Pellet, \\ Enrico Glaab, Alexey Kolodkin, Antonio Raussel, Antony Le Béchec, \\ Stéphane Ballereau, Laurene Meyniel, Isaac Crespo, Hassan Ahmed, \\ Vitaly Volpert, Vincent Lotteau, Nitin Baliga, Leroy Hood, \\ Antonio del Sol, Rudi Balling and Charles Auffray
}

\begin{abstract}
Network analysis is an essential component of systems biology approaches toward understanding the molecular and cellular interactions underlying biological systems functionalities and their perturbations in disease. Regulatory and signalling pathways involve DNA, RNA, proteins and metabolites as key elements to coordinate most aspects of cellular functioning. Cellular processes depend on the structure and dynamics of gene regulatory networks and can be studied by employing a network representation of molecular interactions. This chapter describes several types of biological networks, how combination of different analytic approaches can be used to study diseases, and provides a list of selected tools for network visualization and analysis. It also introduces proteinprotein interaction networks, gene regulatory networks, signalling networks and metabolic networks to illustrate concepts underlying network representation of cellular processes and molecular interactions. It finally discusses how the level of
\end{abstract}

An erratum to this chapter is available at 10.1007/978-94-007-6803-1_19

A. Chaiboonchoe $\cdot$ J. Pellet $\cdot$ S. Ballereau $\cdot$ L. Meyniel $\cdot$ H. Ahmed $\cdot$ V. Volpert

V. Lotteau $\cdot$ C. Auffray $(\bowtie)$

European Institute for Systems Biology and Medicine, CNRS-UCBL-ENS,

Université de Lyon, 50 Avenue Tony Garnier, 69366 Lyon cedex 07, France

e-mail: cauffray@eisbm.org, achaiboonchoe@eisbm.org, jpellet@eisbm.org,

sballereau@eisbm.org, laurene.meyniel@inserm.fr, hahmed@eisbm.org,

vvolpert@eisbm.org, vlotteau@eisbm.org

W. Jurkowski · E. Glaab - A. Kolodkin · A. Raussel · A. Le Béchec · I. Crespo ·

A. d. Sol · R. Balling

Luxembourg Centre for Systems Biomedicine, University of Luxembourg, 7,

Avenue des Hauts-Fourneaux, 4362 Esch-sur-Alzette, Luxembourg

e-mail: wiktor.jurkowski@uni.lu, enrico.glaab@uni.lu, alexey.kolodkin@uni.lu, antonio.raussel@uni.lu, anthony.lebechec@uni.lu, isaac.crespo@uni.lu,

antonio.delsol@uni.lu,rudi.balling@uni.lu

A. Kolodkin · N. Baliga $\cdot$ L. Hood

Institute for Systems Biology, 401 Terry Avenue North, Seattle, WA 98109-5234, USA

e-mail: nbaliga@systemsbiology.org, lhood@systemsbiology.org 
accuracy in inferring functional relationships influences the choice of methods applied for the analysis of a particular biological network type.

Keywords Boolean models • Cell-cell interaction - Continuous models - Database • Discrete models - Diseasome - Gene regulatory networks - Hybrid models • Integrative disease map - Metabolic networks - Multi-scale modeling • Network analysis tools • Network modeling • Network pharmacology • Network topology • Ordinary differential equations $\cdot$ Protein-protein interaction $\cdot$ Signalling networks

\begin{tabular}{|c|c|}
\hline APID & Agile Protein Interaction DataAnalyzer \\
\hline AP-MS & Affinity purification-mass spectrometry \\
\hline ATP & Adenosine triphosphate \\
\hline BioGRID & Biological General Repository for Interaction Datasets \\
\hline CCNA2 & Cyclin-A2 \\
\hline cMap & Connectivity map \\
\hline CYP3A4 & Cytochrome $\mathrm{P}_{450} 3 \mathrm{~A} 4$ \\
\hline CTD & Comparative Toxicogenomics Database \\
\hline DIP & Database of Interacting Proteins \\
\hline DNA & Deoxyribonucleic acid \\
\hline GHEN2PHEN(G2P) & Genotype-To-Phenotype \\
\hline GR & Glucocorticoid receptor \\
\hline GRN & Gene regulatory Network \\
\hline GTP & Guanosine triphosphate \\
\hline hERG & Human Ether-à-go-go-Related Gene \\
\hline HPID & Human Protein Interaction Database \\
\hline HPRD & Human Protein Reference Database \\
\hline HTML & Hyper Text Markup Language \\
\hline IMEx & International Molecular interaction Exchange consortium \\
\hline MIMIx & Molecular Interaction eXperiment \\
\hline MINT & Molecular INTeraction database \\
\hline MIPS & Mammalian Protein-Protein Interaction Database \\
\hline My-DTome & Myocardial infarction drug-target interactome network \\
\hline NR & Nuclear Receptors \\
\hline ODEs & Ordinary Differential Equations \\
\hline OMIM & Online Mendelian Inheritance in Man \\
\hline PDEs & Partial differential equations \\
\hline PHARMGKB & Pharmacogenomics Knowledge Base \\
\hline PPI & Protein-protein interaction \\
\hline PSI-MI & Proteomics Standards Initiative on Molecular Interactions \\
\hline RNA & Ribonucleic acid \\
\hline SBML & Systems Biology Markup Language \\
\hline STRING & Search Tool for the Retrieval of Interacting Genes/Proteins \\
\hline XML & Extensible Markup Language \\
\hline $\mathrm{Y} 2 \mathrm{H}$ & Yeast two-hybrid \\
\hline
\end{tabular}




\subsection{Introduction: From Pathways to Networks}

In a biological pathway of interest, molecular entities such as genes or proteins very often also interact with other entities involved in distinct pathways. Since each pathway represents a specific region of a larger network in a given biological system, network analysis methods can provide additional biological insights that cannot be obtained from pathway analyses alone.

Biological networks comprise nodes that correspond to genes, proteins, metabolites or other biological entities, and edges that correspond to molecular interactions and other functional relationships between the biological entities. In general, in comparison to random networks (network elements connected by chance), biological networks of the same size and connectivity exhibit significant differences in aspects such as: wiring type or presence of topological motifs (groups of inter-connected nodes with a given structure). This affects (1) modularity i.e. the degree of division of the network into subnetworks that comprise densely connected nodes but share few edges outside the module, (2) dissortativity, i.e. the tendency of nodes to connect to other nodes in the network that are associated with different characteristics (e.g. nodes with many connections link to nodes with few connections), and (3) robustness [1] i.e. the resilience of the network to the removal of nodes or edges.

One of the most common strategies used to extract new insights from biological networks is to study the graph topology of a network, i.e. the patterns of interconnections between nodes and edges, based on a key metric: the degree or the number of connections of a node with other nodes. This led to the introduction of the concepts of scale-free networks [2], in which the node degree of connectivity distribution follows a power-law, and of small-world networks [3], in which the distance between nodes grows proportionally to the logarithm of the network size. In the latter, only few nodes act as "highly connected hubs" and the majority of the nodes are of low degree of connectivity (i.e. are engaged in only few interactions) $[4,5]$. This property is believed to confer resistance to random attacks but makes scale-free network extremely susceptible to targeted perturbations [6]. Most biological networks display scale-free properties typical of small-world networks much more often than expected by chance, as is also observed for social networks [7].

These properties make network analysis an interesting approach to study biological systems in order to explain experimental observations and to formulate new hypotheses about biological functions at the molecular, cellular and higher levels of abstraction. Network analysis should however be performed with caution for two reasons. First, most of the networks are largely incomplete, that is they are missing many nodes (e.g. molecules, complexes, phenotypes) and edges (i.e. connections representing reactions, associations or influences). On the other hand, many false positive elements and interactions tend to be included in networks in the absence of contextual information on cellular or tissular localization. Second, the networks have dynamical architectures, i.e. they may change significantly in 
structures during biological or disease processes. There are virtually no examples where connectivity measurements have been made in a dynamical manner [8].

Despite these limitations networks have proven to be valuable tools to represent and analyse complex biological knowledge and processes. The first section of this chapter introduces the basic network types associated with gene regulation, protein-protein interactions, metabolic reactions and signalling processes. Inference of a genetic interaction network from gene co-expression data in the absence of knowledge of the underlying mechanisms, or accurate characterization of chemical reactions with known stoichiometry and kinetic parameters correspond to widely different levels of representation and require distinct computational approaches. The resulting molecular interaction networks need to be integrated with drug and phenotype networks in order to understand perturbations causing and caused by disease, and to facilitate the process of development of adequate medical interventions.

Introduction of network types (Fig. 6.1) is followed by a concise introduction to the main methods available for network topology analysis and modelling approaches: discrete and continuous models and how they can be extended to simulate cell-cell interactions.

In order to understand perturbations causing and caused by disease and to facilitate the process of development of adequate medical interventions, the

(a) Gene Regulatory Network
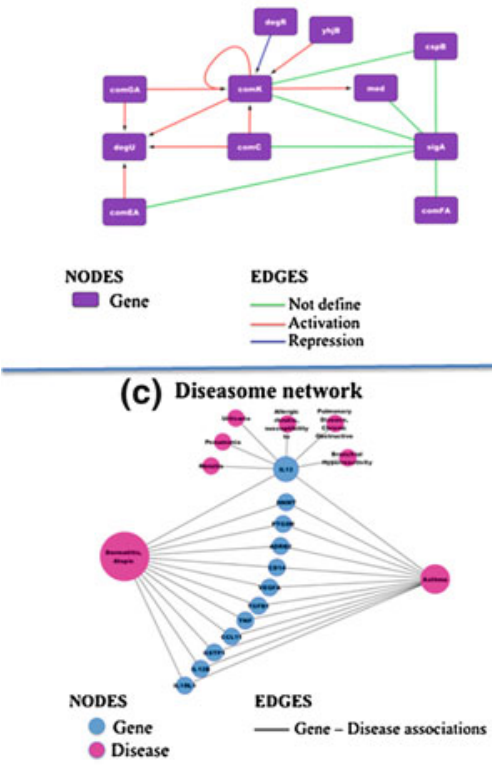

(b) Protein-protein network

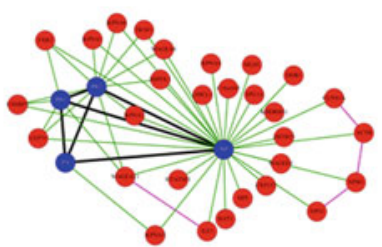

NODES EDGES

Human protein
Viral protein

(d) Integrative disease map

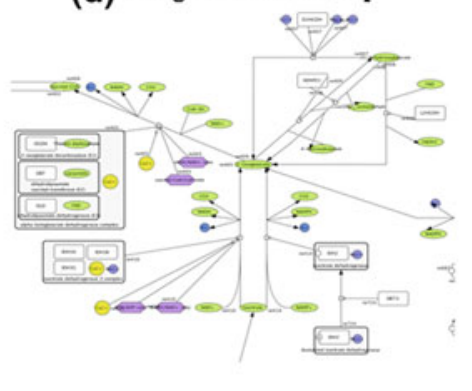

Fig. 6.1 Network types: a gene regulatory network, b protein-protein interaction network, c diseasome network, $\mathbf{d}$ section of an integrative network of disease model 
resulting molecular interaction networks need to be integrated with drug and phenotype networks. We thus present specific examples where knowledge from multiple sources has been integrated to construct network of diseases and drug targets as well as comprehensive disease-focused cellular maps. In the last section we present a short overview of case studies applying network analysis for disease mechanism identification as an illustration of the network pharmacology trend that is now emerging in drug design and development.

\subsection{Network Types}

Among different types of biological networks, this section introduces the most studied molecular network types [9-11]. Although all of them can be reduced to graphs comprising nodes connected by edges, the variety of biological networks arises from the differences in annotated information or purpose for which particular biological knowledge is represented as a network. Thus, the majority of networks discussed here are directed (i.e. an arrow denotes the effect of the source node on the target node) to stress the order of the process, except the proteinprotein interaction network that only combines non-causative pairwise physical interactions without implying succession in time and space. Gene regulatory networks are represented as directed graphs of proteins or small RNA molecules activating or inhibiting gene expression. Therefore a label on the edge orientation displays the regulatory effect. Signalling networks are also directed and signed, and are usually organized to separate processes by cellular localization to better illustrate mutual relationships of particular pathways. The main highlight of metabolic networks is the presence of chemical reactions that extend beyond noncovalent binding. Therefore simple edges need to be complemented with notation of additional substrates and products as well as links representing modulation e.g. activation by enzyme. Because of the complexity of mechanistic description, signalling and metabolic networks are often analysed with fine-tuned continuous models as opposed to typically simpler binary representation of gene regulatory networks.

Lastly, this section extends this simple classification of networks by presenting some examples of disease-related networks.

\subsubsection{Gene Regulatory Networks}

Construction and analysis of a gene regulatory network (GRN) play an important role in understanding the mechanisms of diseases. Recent advances in functional genomics are based on novel experimental and computational approaches that enhance the ability to comprehensively reconstruct the regulatory networks and enrich them with newly discovered components and interactions. 
Gene regulatory networks can be reconstructed from the literature [12] or from experimental data using reverse engineering approaches. These approaches are based on gene expression co-variation patterns inferred from expression or on promoter region occupancy information of specific transcription factors derived from ChIP-Seq or ChIP-Chip experiments [13]. Nevertheless, networks inferred purely from experimental data and those assembled from the literature have different limitations. In the first case, a wealth of data about interactions previously described is ignored. On the other hand, literature-based networks are too disconnected from experimental data to be able to describe input-output relationships, such as cellular responses under specific biological stimuli or mechanisms that determine specific expression patterns. To bridge the gap new methods emerge that combine data from both literature and experiments and provides biological networks contextualized to certain experimental conditions $[14,15]$.

A GRN is commonly represented by a graph usually directed and signed showing interaction (network edges) between components (network nodes) that regulate each other. Because these regulatory components (transcription factors, cofactors, enhancers, repressors or miRNAs) have different properties, a GRN could describe mechanisms of gene regulation at multiple levels (e.g. transcription, post-transcription). Deciphering GRNs from rapidly growing microarray expression databases has been shown to be a very promising approach e.g. in cancer research [16, 17]. Many tools are emerging and have been used for constructing, inferring and analyzing such GRNs. These tools include Boolean networks, Bayesian networks and Ordinary Differential Equations (ODEs) into recently developed web-based applications [18-20]. Considering their complexity, it is often difficult to evaluate or validate the performance of the available tools. In biomedical research, GRNs are expected to improve the current understanding of development and gene interactions in complex systems [21-24].

\subsubsection{Protein-Protein Interaction Networks}

Protein-protein interactions (PPIs) play a vital role in mediating cellular responses in all species and interactome mapping has become an elementary aspect in all areas of systems biology as the scientific community has gathered information on thousands of protein interactions and is increasingly editing, curating and integrating these data sets.

Two complementary ways to obtain comprehensive PPI information exist. The first approach relies on high-throughput experimental methods, including yeast two-hybrid (Y2H) [25, 26], affinity purification followed by mass spectrometry (AP-MS) [27, 28], and luciferase complementation assays [29]. Although recent development of these methods aims at overcoming false-positive discoveries, experimental validation of PPIs by several methods is still crucial. The second approach is to curate all publications in the literature [30], and consult curated datasets from publicly available interaction databases [31]. 
The International Molecular interaction Exchange consortium (IMEx http:// www.imexconsortium.org) [32] comprises eleven databases sharing data curated according to the same common standards. Interactions are reported using the Minimum Information required for reporting a Molecular Interaction eXperiment (MIMIx) [33]. The exchange of information is supported by one major data exchange format: the Proteomics Standards Initiative on Molecular Interactions (PSI-MI) [34] (see Ref. [35] for a complete review) (Table 6.1).

PPI datasets are often visualized as a network. Proteins are represented as nodes, and interactions as connections between nodes. PPI networks are dynamic: they change in time and space to adapt or switch to different physiological conditions.

Various studies have constructed molecular networks with virus proteins to identify their interactions with host proteins and reveal a host-pathogen hybrid protein-interaction network. From a systems biology perspective, a viral infection at the cell level can be viewed as a combination of molecular perturbations allowing viral components production and assembly while generating minor to massive cellular dysfunction. Thus, several large-scale studies of interactions between viral and human proteins have been performed to identify the laws governing virus-host interactomes [36-40]. Taking into account the analytical heterogeneity and the size of the interaction datasets, five corresponding virushuman interactomes were carefully and comprehensively reconstructed from the literature and uniformly analyzed using graph theory as well as structural and functional methods [41].

The systems approach to the biology of viral infection is thus beginning to unravel the global perturbations that lead to viral replication and eventually to pathogenesis. Furthermore, the list of virus-host interactors represents an invaluable resource to derive new molecules, especially for anti-viral therapy.

\subsubsection{Signalling and Metabolic Networks}

Signalling and metabolic networks may be built using a mechanism-based bottomup strategy, with parameters either measured experimentally or assigned arbitrary values in the physiological range. Different questions may then be asked. For example, why is the network organized the way it is? Indeed, at first glance some features in the network may appear paradoxical. However, altering this paradoxical feature in the computer model may disclose design principles underlying the functioning of the network [42]. This approach has for example been successfully applied to design studies of nuclear receptor signalling.

Nuclear Receptors (NR) are proteins that may be activated by signalling molecules (ligands, composed of different intra- and extra-cellular metabolites such as hormones or fatty acids) and then regulate gene expression of their responsive genes. The glucocorticoid receptor (GR) is a NR with an important regulatory role in various cellular functions: gluconeogenesis and glucose uptake, 


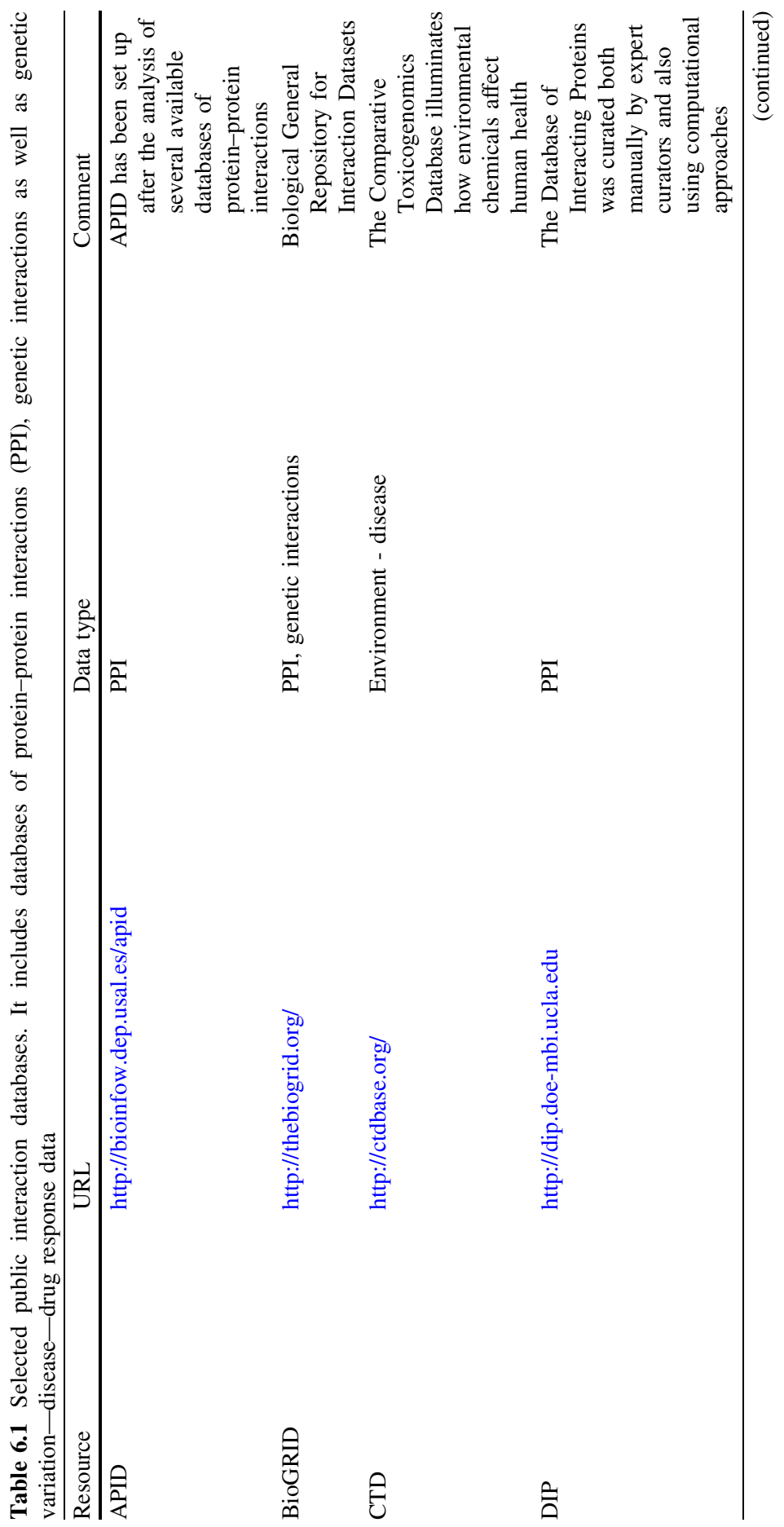




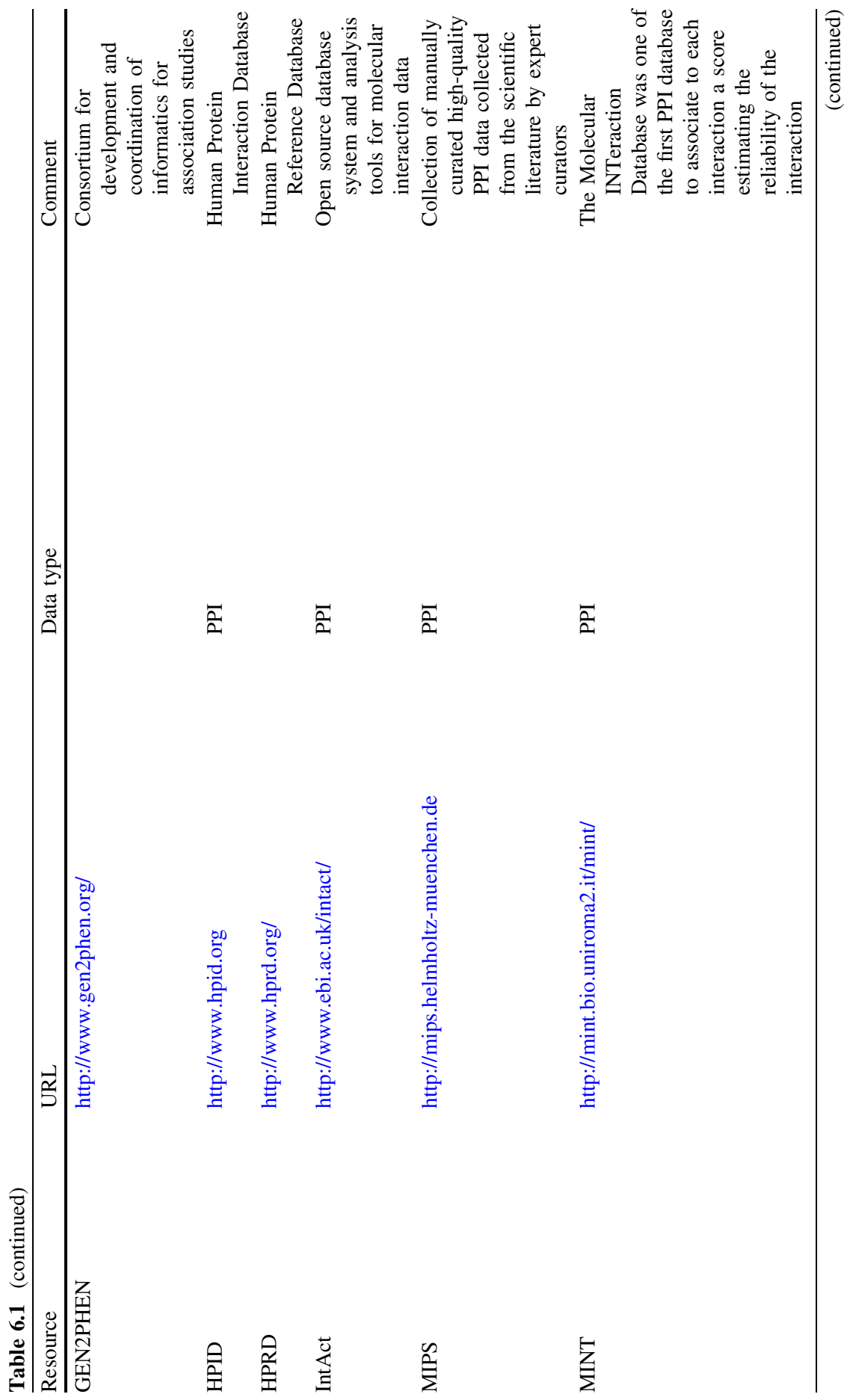




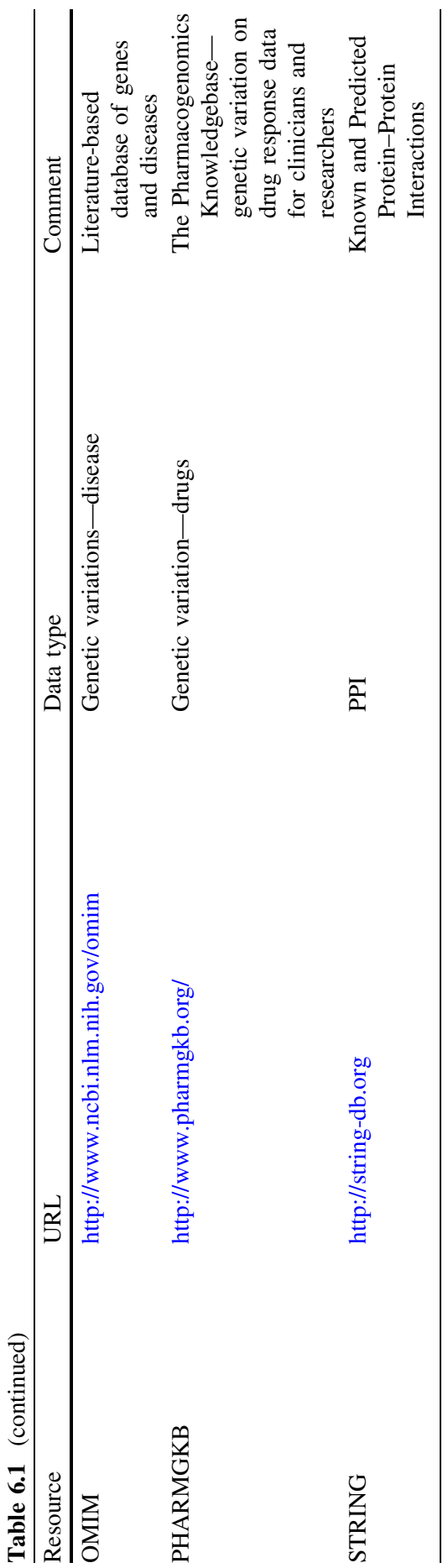


lipolysis in adipose tissues, proteolysis in muscles, osteoblast differentiation and apoptosis [43, 44]. Ligands for GR are steroid hormones such as cortisol. GR has a high rate of nucleo-cytoplasmic shuttling and is predominantly located in the cytoplasm when unbound to a ligand. Upon ligand binding, GR changes its conformation, resulting in its increased affinity to nuclear importins and its decreased affinity to exportins. This causes translocation of the ligand-GR complex to the nucleus, where GR binds to its responsive genes and regulates their transcription $[45,46]$ thereby transmitting signal for gene expression. This network is also metabolic, as it involves nucleo-cytoplasmic transport of the receptor driven by GTP hydrolysis, and ATP and GTP metabolic reactions. Furthermore, concentrations of receptors, importins, exportins and ligand itself (e.g. cortisol continuously degraded by CYP3A4 enzyme) are parts of the larger metabolic network. This is the first paradoxical feature. Why is the receptor not only a receptor? Why does the receptor continuously shuttle between the nucleus and the cytoplasm? The study of GR network showed that nucleo-cytoplasmic shuttling of GR also serves as a smart shuttle for a ligand, which it pumps into the nucleus, thereby increasing the sensitivity and responsiveness of signalling [42].

This example shows that signalling and metabolic networks should not be analyzed separately, but instead be integrated together and with regulatory networks. This integration has recently become an important topic in systems biology [47].

\subsubsection{Integrative Approaches Applied to Human Diseases}

Disease networks can be viewed as networks of associations between diseasecausing mutations and diseases or as high-resolution interaction maps integrating metabolic reactions, signalling pathways and gene regulatory networks.

The link between all genetic disorders (the human disease phenome) and the complete list of disease genes (the disease genome) results in a global view of the "diseasome", i.e. the combined set of all known disease gene associations $[11,48]$. Here diseases form a network in which two diseases are connected if they share at least one gene. In the disease gene network, diseases or genes are represented as nodes and gene-disorder association as edges. In such a network representation, obesity, for example, is connected to at least seven other disorders such as diabetes, asthma, and insulin resistance because genes associated with these diseases are known to affect obesity as well [7]. In recent years several disease map projects have flourished, such as the pioneering work at the Systems Biology Institute (Okinawa, Japan) that was a hub of collaborative, community-based efforts to reconstruct a map of tuberculosis [49]. In addition, the Connectivity map (cMap) [50] developed by the Broad Institute aims to create a map connecting genes, diseases and drugs using a repository of gene expression profiles to represent different biological states including gene alterations and disease phenotypes. cMap is a web tool with preloaded data in which query results can be interpreted by strong (positive or negative) connection or absence of connection [50]. 
These efforts would be not possible without integrating publically available disease-related knowledge. Online Mendelian Inheritance in Man (OMIM) is a catalogue of human genes and genetic disorders and traits that has been updated continuously for several decades [51]. As of May 2012, it contained 2,795 diseases genes and 4,669 disorders for which the molecular basis is known. Other databases, including the Pharmacogenomics Knowledge Base (PHARMGKB) [52] or the Comparative Toxicogenomics Database (CTD), focus on different aspects of phenotype-genotype relationships. GEN2PHEN (G2P) is a European project aiming at gathering and curating information to build a knowledgebase of genotype-phenotype interactions [53]. This project will build a linked database from existing publicly accessible databases and integrate all available data using highperformance analytical tools.

The human cancer map project is exploiting the idea that network motifs can contribute to a network switching from one stable state to another [54]. Analyzing networks reconstructed from microarray experiments and molecular interaction maps, authors identified genes participating in bi-stable switches i.e. network motifs that can exist in two stable states and drive the change of the network states. Expression states of genes within bi-stable switches were compared between hepatocellular carcinoma or lung cancer and healthy control samples. In both cases, bi-stable switches made of differentially expressed genes were proposed to be a network mechanism for locking in disease states. Such studies have identified two important hubs: cyclins and albumin. In hepatocellular carcinoma, up-regulation of CCNA2 (cyclin A2) leads to changes in expression of downstream genes, in accordance with the general observation that perturbations of oscillations in cyclins concentrations can have a detrimental effect on cell development. For instance, ubiquitination of cyclin A1 induces apoptosis via activation of caspase-3 [55] or cyclin D1 degradation activated by a troglitazone derivative [56]. Also, the lack of phosphorylation of cyclin E, due to mutations, results in its increased stability, which has implications for breast cancer [57]. Another important hub is the up-regulated albumin gene. Albumin is a large transport molecule with an adaptable two-domain structure that can bind an array of lipids, peptides, metabolites and drugs. Allosteric modulation of albumin may change its binding and cargo transport properties, and hence directly affect downstream cellular processes. For example, electron spin resonance studies of albumin modulation by cancer-related small molecule markers revealed significant differences in binding of albumin-specific 16-doxyl-stearic acid probe. During disease progression the albumin pool saturates with cancer cellular metabolites, thus indicating an affected albumin state [58].

\subsection{Network Analysis}

Many software tools are available to reconstruct biological networks from experimental data and then position nodes on a graph according to a topological placement algorithm. This visual network of interconnected nodes can then be 


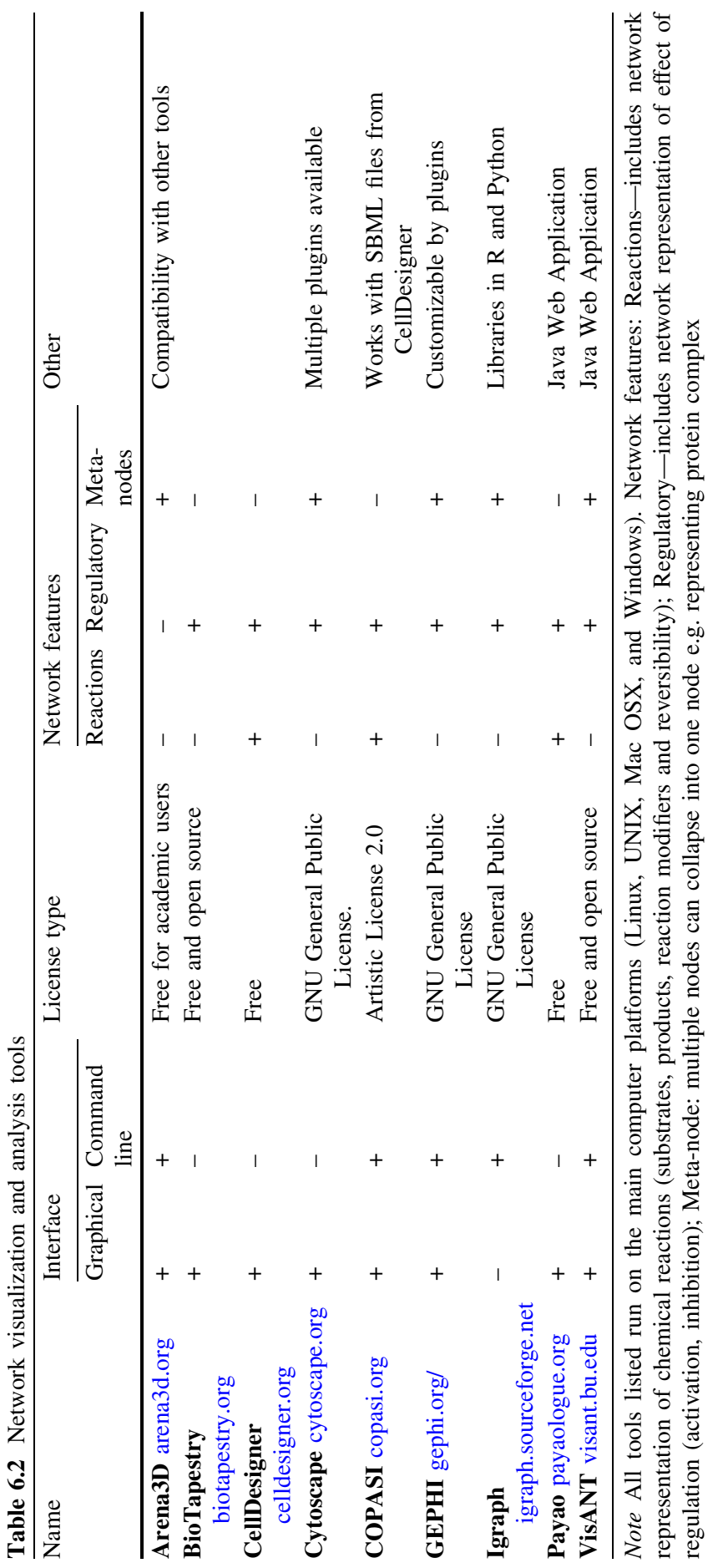


transformed into various mathematical models e.g. flux balance models, kinetic ODE models or space Partial Differential Equations (PDEs) models, which can be fitted to experimental data and used to simulate the kinetic behaviour of biological networks. Since each of the many different tools available only performs one of those tasks, designing an integrated and efficient analysis pipeline is challenging. Such difficulties prompted the development of a single unified standard language suitable for the interchange between various tools: the systems biological markup language (SBML) [59]. SBML is based on the widely used Extensible Mark-up Language (XML), allows the development of graphical interfaces and analysis frameworks to display and analyze interaction maps. It is therefore becoming a standard for the representation and annotation of biological processes. The following chapter discusses several tools and the perspectives of their future development.

\subsubsection{Network Analysis Tools}

Among public network management tools that currently exist to visually explore and analyse biological networks (see review in [60]) such as Arena3D [61], GEPHI [62], igraph [63] and VisANT [64] (Table 6.2), Cytoscape [65], CellDesigner [66] and Copasi [67] are the most powerful and widely used.

Cytoscape (449,030 downloads as on September 2012) is the most popular software for the visualization and analysis of interaction networks. Its functionality can be extended using the collection of plugins developed by the expanding Cytoscape community of users. Recently Cytoscape web [68] became available to embed interactive networks in an HTML page.

CellDesigner $(65,105$ downloads as on September 2012) is a structured diagram editor for drawing integrative maps (including gene regulatory and biochemical networks) that define reactions and interactions between various types of biochemical species (genes, proteins, small molecules) in the context of their subcellular localization and in relation to the biological or pathological processes in which they are involved.

Copasi (26,000 downloads as on January 2012) is a stand-alone program that supports models in the SBML standard and can simulate their behavior using ODEs or Gillespie's stochastic simulation algorithm.

The growing popularity of Cell Designer and Copasi stems from their compatibility and complementarity. The strength of Cell Designer is its easy-use interface for the drawing of biochemical networks while that of Copasi is its convenience for fitting the model to experimental data, metabolic control analysis and dynamic simulations. The network diagram and mathematical model created in Cell Designer can be easily transformed into Copasi and vice versa. 


\subsubsection{Network Topology Analysis}

The discovery that many real-world biological networks exhibit scale-free and small-world properties [69, 70] has led to a surge of new topological analysis methods for biological networks. The study of global topological properties enables a general characterization of a network, e.g. providing information on its robustness to perturbations. In contrast, analysis of local topological properties can provide specific insights on single nodes (e.g. on their centrality in the network and their tendency to form dense clusters with other nodes), which can also be exploited in high-throughput data analysis applications. A comprehensive and detailed discussion of network topological properties has been compiled recently in a book dedicated to this topic [71].

Molecular interaction networks are assembled from public interaction databases like BioGRID [72], HPRD [73], IntAct [74], MIPS [75], DIP [76], HPID, [77] MINT [78], or meta-databases such as APID [79]. Several issues affect the quality of assembled networks and other integration tasks, e.g. false positives in the input data sources and incomplete lists of interactions. Commonly used pre-processing methods filter collected interactions using a combined set of criteria, e.g. the number and type of experiments that were used to verify an interaction and data source-specific confidence scores. One of the most comprehensive collections of molecular interaction data for different species is provided by the STRING database [80], which also contains different types of confidence scores for each interaction to filter the data. Since many network analysis methods require a single connected component as input, a final pre-processing step often involves removing small, disconnected components from a graph representation of the assembled interactions.

Regarding the typical applications for topological analyses, global descriptors are mainly used for the general characterization of large-scale biological networks. Since these global network properties have already been studied extensively for several biological network types and species, the corresponding analyses are only likely to provide new insights when studying a novel network type. However, new applications for employing global topological analyses as components of other algorithms have been proposed recently, e.g. to improve the generation of gene co-expression networks by analysing the scale-free property for tentative networks [81]. By contrast, local topological network characteristics have already been exploited by a wide variety of new data mining approaches recently, including methods to identify dense communities [82] of nodes [83, 84], methods to compare mapped gene and protein sets in terms of their network topological properties [85], and approaches to score distances between nodes for prioritizing disease genes [86]. Interestingly, recent studies have shown that cancer-associated genes tend to have outstanding topological characteristics [87], even when accounting for study-specific biases, and that topological information can facilitate cancer classification [88]. 
In summary, topological characteristics of complete biological networks, subgraphs and single nodes provide a valuable information source for the integrated analysis of functional genomics data. Network topology analyses are often combined with graph-theoretic methods to identify dense communities or clusters of nodes [83, 84], or to quantify the similarity between single nodes or node sets using different network-based distance measures [86, 89-91]. However, topological properties can also be exploited in other domains, e.g. as features in machine learning methods for clustering and prediction [92], as part of scoring criteria in de novo pathway prediction [93], and to evaluate the stability and integrity of biological networks generated from combined microarray correlation analysis and literature mining [94].

\subsubsection{Network Modeling}

Various approaches describe biological networks mathematically. The simplest is to build a discreet model based on graph theory. In this approach, each node (e.g. molecule) of the biological network may be present in two (e.g. 0 or 1) or several fixed states. Each state affects interactions of a node with other nodes differently and the underlying mechanisms need not be known. In contrast, continuous modeling offers alternative approaches that account for the subtle gradual changes in the concentration of species in a biological network. Three main types of continuous models exist: a continuous 'microscopic' model traces every molecule individually while a 'mesoscopic' model uses stochastic simulations with molecular concentrations described in terms of probability functions; lastly, a 'macroscopic' model neglects limitations in the diffusion of molecules on the reaction rate, considers each species of biomolecules as a single pool described with a system of ODEs. The latter is a very popular approach to model intracellular metabolic networks in which the number of molecules is rather high and could be viewed as a single pool with a certain mean concentration. Another important question relates to interactions between different cells. This requires special cellcell interaction models. In this section we will discuss three main modeling approaches: discrete modeling, continuous modeling based on ODEs and modeling of cell-cell interactions.

\subsubsection{Discrete Models}

In the Boolean framework, the state of each node in the network is conceptually described as being active (represented by ' 1 ') or inactive (represented by ' 0 '). Similarly, directed edges in the network are represented as activators or inhibitors. The state of each node is therefore determined by the states of the nodes that activate and/or inhibit them, following predefined logic rules inferred from experimental data and/or expert knowledge. Boolean models provide an abstraction of genetic circuits 
that, despite being simplistic, are able to capture important aspects of cell development [95]. Importantly, they enable the study of key dynamical properties such as steady states, defined as stable states of the network that might be stationary or oscillatory. In a Boolean model, the consequences in the global system of a given perturbation (represented as a change in the state of a node) are assessed through updating the states of the nodes in the network following the logic functions. The updating process can be done synchronously or asynchronously, depending on whether all nodes are updated simultaneously or in a step-wise manner, respectively $[96,97]$. Indeed, the steady states reached following these two strategies might be different, and it is usually recommended to combine their results. Boolean networks allow integration of qualitative information into the modelling process and have been successfully applied to many relevant biological systems [6, 96, 98]. Despite being deterministic, the Boolean framework allows inclusion of stochastic components in the models, either in the states of nodes [99, 100] or in the logic functions [101]. Together with Boolean models, multiple valued logic models [97, 102] are another type of discrete logic models that allow considering more than two levels, e.g. low, medium or high expression, which might be more realistic, but is associated with a higher computational cost.

Discrete logic models have the fundamental advantage over continuous models such as ODEs that they can use qualitative data to build a gene regulatory network. The amount and availability of qualitative information is larger than the quantitative parameters required in ODEs. However, discrete logic approaches cannot model the evolution in time of the quantitative concentrations of the species in the system. To bridge the gap between ODEs and discrete logic models, a third category of approaches has been developed, where the initial discrete logic network is transformed into a system of ODEs, following different strategies with successful results [98, 103-105].

\subsubsection{Continuous Models}

ODEs are often used for dynamic modelling of regulatory networks of different levels of complexity from bacteria [106] to eukaryotes [107-110]. Changes in concentrations of each species in the network of interacting biomolecules may be expressed through balance equations and rate equations. In this framework, variations in concentrations of molecules as a function of time may be represented by differential equations establishing the stoichiometry and the reaction rates of the transformations and/or transport events [111, 112]. These equations contain the information about component properties for each molecule. Integrating all ODEs together enables reconstruction of the emergent behaviour of a whole system, e.g. simulation of its dynamics in silico.

In order to validate a dynamic model, the simulated systemic behavior is compared with the behaviour of a real system; this comparison is especially powerful if both the real object and its model are challenged with a series of perturbations that were not considered while the model was under construction nor 
used for parameter fitting. In many cases the predicted behaviour does not fit that of the actual biological system. This may then lead to the discovery of mechanisms missing or poorly described in the model. For example, the yeast glycolysis model built by Teusink and co-authors predicted that yeasts would invest too much of ATP in the first ATP-consuming reactions, and then die from the accumulation of these compounds and from a deficit of phosphate. The observation that yeasts are more robust in reality than in silico, provoked re-thinking of the model mechanisms and led to the discovery of an additional negative feedback loop which regulates the first phosphorylation step of glycolysis and prevents the turbo explosion in ATP-consuming reactions [113].

Once a model simulates the biological system behaviour adequately, it can be used for various goals. For example, using a in silico cell model of a metabolic network, one can design modification of the organism metabolism: e.g. the metabolism of Escherichia coli can be modified in such a way that E. coli produces polylactic acid - a biopolymer analogous to petroleum-based polymers which can be used in industry [114]; the metabolism of insects can be modified to make insects a promising source of food to meet the challenge of providing the protein supply to feed over 9 billion humans in the near future [115]. Cell models have become useful in differential network-based drug discovery: a kinetic model of the known metabolic network may help to find proper target enzymes either for correcting malfunctioning of a human cell or for killing a cancer cell [116] or a parasite [117]. For instance, comparison between glycolysis in Trypanosoma brucei (parasite causing African trypanosomiasis in humans), and glycolysis of human erythrocytes was used for the development of drugs killing T. brucei with reduced side effects [118].

The main drawback of in silico cell models is that they usually require knowledge of the mechanisms of interactions and estimates of numerous parameters for these interactions, which are only available for few systems that are very well characterized experimentally. However one may anticipate that tremendous progress in functional genomics, proteomics, metabolomics and bioinformatics should help to obtain the lacking information in the near future.

\subsubsection{Cell-Cell Interactions and Multi-Scale Modeling}

Intracellular regulatory networks are used for the analysis of a single cell or of a population of identical cells in the same state. However, different cell types usually exist in the same tissue while cells of the same type can have different concentrations of intracellular proteins due to intrinsic stochastic variations or to different cellular environments. Their interaction can influence intracellular regulatory networks e.g. through bistable switches in the network. Hence, local regulation by cell-cell interaction within the tissue and global regulation through interactions with other organs should both be taken into account in the analysis of regulatory networks. Multi-scale modeling therefore includes intracellular and molecular levels as well as interactions at the levels of cell populations and of other tissues and organs. 
Intracellular regulation of individual cells and their local and global interactions can be studied with hybrid models in which cells are considered as individual objects, intracellular regulatory networks are described by ODEs or by Boolean networks, and the extracellular matrix together with nutrients, hormones and other signalling molecules by PDEs. Hybrid models can also account for natural stochastic variations of intracellular concentrations, cell motion, cell proliferation, differentiation and apoptosis. They are well adapted to the representation and analysis of various biological systems and biomedical situations. They can include the pharmacokinetics of medical treatments, prediction and optimization of their efficacy. However, they require a detailed knowledge of intracellular and extracellular regulations and sophisticated modeling tools [119, 120].

A hybrid model of erythropoiesis and leukemia treatment was described recently [121]. Erythropoiesis, or red blood cell production, occurs mainly in the bone marrow in small units called erythroblastic islands. They contain, on average, several dozens of cells in different phases of cell differentiation: erythroid progenitors, erythroblasts, reticulocytes structured around a macrophage. Normal functioning of erythropoiesis depends on the balance between proliferation, differentiation and apoptosis of erythroid progenitors. The intracellular regulatory network in erythroid progenitors, described by ODEs, determines cell fate due to bistability, where one stable equilibrium corresponds to proliferation without differentiation and another one to differentiation/apoptosis. The choice between these stable equilibria and, at the next stage, between differentiation and apoptosis is determined by two factors: local extracellular regulation, e.g. Fas-ligand produced by more mature cells and growth factors produced by macrophages; and global regulation by hormones, such as erythropoietin, with concentrations depending on the total number of erythrocytes produced by erythroblastic islands. Biochemical substances in the extracellular matrix influence intracellular regulation through the coefficients of the ODE system while their concentration is described by PDEs. This multi-level modeling simulates erythropoiesis in normal and stress situations in agreement with experimental data. It also explains the role of central macrophages in controlling erythroblastic islands. Strong perturbations of the system caused for example by mutations or dysfunction of regulatory mechanisms can result in various blood diseases such as anemia or leukemia.

\subsubsection{Network Analysis for Systems Biomedicine and Pharmacology}

\subsubsection{Network Analysis of Disease}

Network analysis may be also a valuable approach to study mechanisms underlying pathology and disease susceptibility. For instance studies of network dynamics can shed light on disease-related network states. Different stable steady states appear to be related to distinct phenotypic states of the cell [122]. 
Robustness of biological networks allows maintenance of a certain phenotypic state over a range of perturbations and may play an important role in controlling state transitions when such stable states are reached. It has been postulated that network circuits displaying bi- and multi-stability may drive network state transitions associated to disease progression and then maintain networks in diseased states [123]. In particular, bi-stable switches in protein-protein interaction or regulatory networks allow cells to enter into irreversible paths and assume different fates depending on which genes are expressed or silent [124, 125].

An analysis of pathological response to fat diet [126] or the mechanism of prion disease [127] using Boolean modelling recently showed the importance of network motifs in stabilizing the disease-related network state. Indeed circuits regulating bi-stability do not function in isolation but are assembled as an interconnected core cluster of genes that regulate one another thereby ensuring the stability of the network. In addition, differentially expressed genes involved in bi-stable switches are central to the regulatory network and can thus efficiently propagate perturbations to more distant regions of the network. These concepts are supported by previous studies focusing on network motifs to show the key role of network bi-stable feedback loops in cell fate determination and plasticity [128-130], and the implication of bi-stable circuits in the resilience and progression of human cancers, where the healthy and cancer states are considered to be the two stable states $[131,132]$.

\subsubsection{Network Pharmacology}

In a typical drug development approach, an active compound is optimized to act on a single protein target and other potential interactions are considered only to increase specificity of binding to a given receptor subtype while avoiding known toxic effects. This view has been recently challenged in the field of polypharmacology as it is recognized that drugs can effectively act on multiple targets, e.g. the recent discovery of the simultaneous inhibition of two families of oncogenes (tyrosine and phosphoinositide kinases) by the same effector [133]. Studies of offtarget effects can lead to successful drug repurposing [134, 135] or to the prevention of adverse side effects. For instance, blocking of the hERG potassium channel is responsible for many severe drug-induced cardiac arrhythmias and is therefore included as a part of safety testing in drug development [136].

On the other hand, a single drug is unlikely to be sufficient to target the multiple facets of pathological processes. Rational drug design is now attempting to define mixtures of bioactive compounds that constitute drugs often exerting synergistic therapeutic effects, as in the long tradition of herbal medicine [137].

Systems biology approaches are used to develop tools necessary to understand complex drug-target relationships. Network pharmacology is integrating information on diseases, targets, and drugs with biological data to infer networks of drug targets, disease-related genes or drug-disease interactions [138-140]. Properties of such networks may help in understanding individual drug response due to 
changing genetic background [122], or contribute to the discovery of new druggable targets, therapeutic strategies to overcome adverse drug effects. In some cases, these studies are accompanied with development of resources and tools tailored for medical applications. My-DTome [141] is an example of a web-based searchable resource on drug-target interactome networks relevant to myocardial infarction.

\subsection{Conclusions}

Network analysis to organize and mine biological knowledge has become an inherent element of computational systems biology. By focusing on certain aspects of biochemical processes in living cells, the network may represent gene regulatory, metabolic, signalling processes and connect network elements with functional associations or, when used without imputing causality, represent physical binding of molecules. To answer specific biological questions different methodologies should be considered depending on the completeness of description that is accessible. This chapter presented an overview of approaches used to derive meaningful conclusions from graph topology, and develop simulations of network states using discrete and continuous models. The use of these approaches may be extended to simulate processes on higher (cell-cell interactions) levels of organization or combined to represent multiple levels from molecules to organs. The study of disease-related networks is increasingly impacting identification of drug targets with limited adverse effects, triggering the emergence of network pharmacology.

Acknowledgments This work was supported by the CNRS, and in part by the EU grants to CA in the context of the U-BIOPRED consortium (Unbiased Biomarkers for the PREDiction of respiratory disease outcomes, Grant Agreement IMI 115010) and the MeDALL consortium (Mechanisms of the Development of Allergy, Grant Agreement FP7 N 264357). The formation of the European Institute for Systems Biology \& Medicine hosted at Claude Bernard University is supported by the Lyonbiopole competitive cluster and its academic, industrial and local authority partners, including Grand Lyon, Région Rhône-Alpes, Direction de la Recherche et de la Technologie, and the Finovi Foundation (CA).

We would like to acknowledge the support of the Luxembourg Centre for Systems Biomedicine and the University of Luxembourg (LH and RB), the NIH General Medical Sciences Center for Systems Biology GM076547 (LH) and a Department of Defense contract on Liver Toxicity W911SR-09-C-0062 (LH).

\section{References}

1. Callaway DS, Newman MEJ, Strogatz SH, Watts DJ (2000) Network robustness and fragility: percolation on random graphs. Phys Rev Lett 85:5468-5471

2. Barabási AL, Albert R (1999) Emergence of scaling in random networks. science 286, 509

3. Watts DJ, Strogatz SH (1998) Collective dynamics of 'small-world'networks. Nature 393:440-442 
4. Barabási A-L, Oltvai ZN (2004) Network biology: understanding the cell's functional organization. Nat Rev Genet 5:101-113

5. Barabási A-L (2009) Scale-free networks: a decade and beyond. Science 325:412-413

6. Albert R, Jeong H, Barabasi AL (2000) Error and attack tolerance of complex networks. Nature 406, 378-382

7. Barabási A-L (2007) Network medicine-from obesity to the 'diseasome'. N Engl J Med 357:404-407

8. Ideker T, Krogan NJ (2012) Differential network biology. Mol Syst Biol 8

9. Vidal M, Cusick ME, Barabási A-L (2011) Interactome networks and human disease. Cell 144:986-998

10. Pavlopoulos $\mathrm{G}$ et al (2011) Using graph theory to analyze biological networks. BioData Mining 4:10

11. Barabási A-L, Gulbahce N Loscalzo J (2011) Network medicine: a network-based approach to human disease. Nat Rev Genet 12, 56-68

12. Bauer-Mehren A, Furlong LI, Sanz F (2009) Pathway databases and tools for their exploitation: benefits, current limitations and challenges. Mol Syst Biol 5:290

13. Johnson DS, Mortazavi A, Myers RM, Wold B (2007) Genome-wide mapping of in Vivo protein-DNA interactions. Science 316:1497-1502

14. Crespo I, Krishna A, Le Béchec A Del Sol A (2012) Predicting missing expression values in gene regulatory networks using a discrete logic modeling optimization guided by network stable states. Nucleic Acids Res doi:10.1093/nar/gks785

15. Saez-Rodriguez J et al (2009) Discrete logic modelling as a means to link protein signalling networks with functional analysis of mammalian signal transduction. Mol Syst Biol 5:331

16. Madhamshettiwar P, Maetschke S, Davis M, Reverter A, Ragan M (2012) Gene regulatory network inference: evaluation and application to ovarian cancer allows the prioritization of drug targets. Genome Med 4:41

17. Zhang Y, Xuan J, De los Reyes BG, Clarke R Ressom HW (2010) Reconstruction of gene regulatory modules in cancer cell cycle by multi-source data integration. PLoS ONE 5, e10268

18. Hache H, Lehrach H Herwig R (2009) Reverse engineering of gene regulatory networks: a comparative study. EURASIP J Bioinf Syst Biol, 8:1-8:12

19. Marbach D et al. (2010) Revealing strengths and weaknesses of methods for gene network inference. In: Proceedings of the national academy of sciences 107, 6286-6291

20. Haibe-Kains B et al (2011) Predictive networks: a flexible, open source, web application for integration and analysis of human gene networks. Nucleic Acids Res 40:D866-D875

21. Cooke EJ, Savage RS, Wild DL (2009) Computational approaches to the integration of gene expression, ChIP-chip and sequence data in the inference of gene regulatory networks. Semin Cell Dev Biol 20:863-868

22. Nazri A, Lio P (2012) Investigating meta-approaches for reconstructing gene networks in a mammalian cellular context. PLoS ONE 7:e28713

23. Ahmad FK, Deris S, Othman NH (2011) The inference of breast cancer metastasis through gene regulatory networks. J Biomed Inform. doi:10.1016/j.jbi.2011.11.015

24. Davidson EH (2010) Emerging properties of animal gene regulatory networks. pp 911-920

25. Fields S, Song O (1989) A novel genetic system to detect protein-protein interactions. Nature 340:245-246

26. Parrish JR, Gulyas KD, Finley RL Jr (2006) Yeast two-hybrid contributions to interactome mapping. Curr Opin Biotechnol 17:387-393

27. Rigaut $G$ et al (1999) A generic protein purification method for protein complex characterization and proteome exploration. Nat Biotechnol 17:1030-1032

28. Köcher T, Superti-Furga G (2007) Mass spectrometry-based functional proteomics: from molecular machines to protein networks. Nat Methods 4:807-815

29. Cassonnet $P$ et al (2011) Benchmarking a luciferase complementation assay for detecting protein complexes. Nat Methods 8:990-992

30. Roberts PM (2006) Mining literature for systems biology. Brief. Bioinformatics 7:399-406 
31. Lehne B, Schlitt T (2009) Protein-protein interaction databases: keeping up with growing interactomes. Hum. Genomics 3:291-297

32. Orchard S et al (2012) Protein interaction data curation: the International Molecular Exchange (IMEx) consortium. Nat Methods 9:345-350

33. Orchard $S$ et al (2007) The minimum information required for reporting a molecular interaction experiment (MIMIx). Nat Biotechnol 25:894-898

34. Hermjakob $\mathrm{H}$ et al (2004) The HUPO PSI's molecular interaction format-a community standard for the representation of protein interaction data. Nat Biotechnol 22:177-183

35. Orchard S, Kerrien S (2010) Molecular interactions and data standardisation. Methods Mol Biol 604:309-318

36. De Chassey B et al (2008) Hepatitis C virus infection protein network. Mol Syst Biol 4:230

37. Zhang L et al (2009) Analysis of vaccinia virus-host protein-protein interactions: validations of yeast two-hybrid screenings. J Proteome Res 8:4311-4318

38. Calderwood MA et al. (2007) Epstein-Barr virus and virus human protein interaction maps. Proc Natl Acad Sc. USA. 104, 7606-7611

39. Shapira SD et al (2009) A physical and regulatory map of host-influenza interactions reveals pathways in H1N1 infection. Cell 139:1255-1267

40. Khadka S et al. (2011) A physical interaction network of dengue virus and human proteins. Mol. Cell Proteomics 10, M111.012187

41. Meyniel-Schicklin L, De Chassey B, Andre P, Lotteau V (2012) Viruses and interactomes in translation. Mol Cell Proteomics: MCP. doi:10.1074/mcp.M111.014738

42. Kolodkin AN et al (2010) Design principles of nuclear receptor signaling: how complex networking improves signal transduction. Mol Syst Biol 6:446

43. Eijken M et al (2006) The essential role of glucocorticoids for proper human osteoblast differentiation and matrix mineralization. Mol Cell Endocrinol 248:87-93

44. Zhou JG, Cidlowski JA (2005) The human glucocorticoid receptor: one gene, multiple proteins and diverse responses. Steroids 70:407-417

45. Cutress ML, Whitaker HC, Mills IG, Stewart M, Neal DE (2008) Structural basis for the nuclear import of the human androgen receptor. J Cell Sci 121:957-968

46. Heitzer MD, Wolf IM, Sanchez ER, Witchel SF, DeFranco DB (2007) Glucocorticoid receptor physiology. Rev Endocr Metab Disord 8:321-330

47. Lee JM, Min Lee J, Gianchandani EP, Eddy JA, Papin JA (2008) Dynamic analysis of integrated signaling, metabolic, and regulatory networks. e1000086

48. Goh K-I et al (2007) The human disease network. Proc Natl Acad Sci USA 104:8685-8690

49. Kitano H, Ghosh S, Matsuoka Y (2011) Social engineering for virtual 'big science' in systems biology. Nat Chem Biol 7:323-326

50. Lamb J (2007) The connectivity map: a new tool for biomedical research. Nat Rev Cancer 7:54-60

51. Hamosh A, Scott AF, Amberger J, Valle D, McKusick VA (2000) Online Mendelian Inheritance in Man (OMIM). Hum Mutat 15:57-61

52. Klein TE et al (2001) Integrating genotype and phenotype information: an overview of the PharmGKB project. Pharmacogenetics research network and knowledge base. Pharmacogenomics J 1:167-170

53. Webb AJ, Thorisson GA, Brookes AJ (2011) An informatics project and online 'Knowledge Centre' supporting modern genotype-to-phenotype research. 543-550

54. Shiraishi T, Matsuyama S, Kitano H (2010) Large-scale analysis of network bistability for human cancers. PLoS Comput Biol 6:e1000851

55. Ekberg J, Persson JL (2009) Post-translational modification of cyclin A1 is associated with staurosporine and TNFalpha induced apoptosis in leukemic cells. Mol Cell Biochem 320:115-124

56. Wei S et al (2008) A novel mechanism by which thiazolidinediones facilitate the proteasomal degradation of cyclin D1 in cancer cells. J biol chem 283:26759-26770

57. Mull BB, Cox J, Bui T, Keyomarsi K (2009) Post-translational modification and stability of low molecular weight cyclin E. Oncogene 28:3167-3176 
58. Gurachevsky A, Muravskaya E, Gurachevskaya T, Smirnova L, Muravsky V (2007) Cancer-associated alteration in fatty acid binding to albumin studied by spin-label electron spin resonance. Cancer Invest 25:378-383

59. Hucka $\mathrm{M}$ et al (2003) The systems biology markup language (SBML): a medium for representation and exchange of biochemical network models. Bioinformatics 19:524-531

60. Sanz-Pamplona R et al (2012) Tools for protein-protein interaction network analysis in cancer research. Clin Transl Oncol 14:3-14

61. Pavlopoulos GA et al (2008) Arena3D: visualization of biological networks in 3D. BMC Syst Biol 2:104

62. Bastian M, Heymann S, Jacomy Gephi M (2009) An open source software for exploring and manipulating networks

63. Csardi G, Nepusz T (2006) The igraph software package for complex network research. Int J Complex Syst Complex Sy, 1695

64. Hu Z et al (2009) VisANT 3.5: multi-scale network visualization, analysis and inference based on the gene ontology. Nucleic Acids Res 37:W115-W121

65. Shannon P et al (2003) Cytoscape: a software environment for integrated models of biomolecular interaction networks. Genome Res 13:2498-2504

66. Funahashi A et al. (2008) CellDesigner 3.5: A versatile modeling tool for biochemical networks. Proc IEEE 96, 1254-1265

67. Hoops S et al (2006) COPASI-a complex pathway simulator. Bioinformatics 22:3067-3074

68. Lopes CT et al (2010) Cytoscape web: an interactive web-based network browser. Bioinformatics 26:2347-2348

69. Wuchty S (2001) Scale-free behavior in protein domain networks. Mol Biol Evol 18:1694-1702

70. Böde C et al (2007) Network analysis of protein dynamics. FEBS Lett 581:2776-2782

71. Junker BH, Schreiber F, Ebrary I (2008) Analysis of biological networks. (Wiley online library)

72. Stark C et al (2006) BioGRID: a general repository for interaction datasets. Nucleic Acids Res 34:D535-D539

73. Peri $\mathrm{S}$ et al (2004) Human protein reference database as a discovery resource for proteomics. Nucleic Acids Res 32:D497-D501

74. Hermjakob H et al (2004) IntAct: an open source molecular interaction database. Nucleic Acids Res 32:D452-D455

75. Mewes HW et al (2002) MIPS: a database for genomes and protein sequences. Nucleic Acids Res 30:31-34

76. Xenarios I et al (2002) DIP, the database of interacting proteins: a research tool for studying cellular networks of protein interactions. Nucleic Acids Res 30:303-305

77. Han K, Park B, Kim H, Hong J, Park J (2004) HPID: the human protein interaction database. Bioinformatics 20:2466-2470

78. Zanzoni A et al (2002) MINT: a molecular interaction database. FEBS Lett 513:135-140

79. Prieto C, De Las Rivas J (2006) APID: agile protein interaction dataanalyzer. Nucleic Acids Res 34, W298-302

80. Szklarczyk D et al (2011) The STRING database in 2011: functional interaction networks of proteins, globally integrated and scored. Nucleic Acids Res 39:D561-D568

81. Zhang B, Horvath S (2005) A general framework for weighted gene co-expression network analysis. Stat Appli Genet Mol Biol 4:17

82. Radicchi F, Castellano C, Cecconi F, Loreto V, Parisi D (2004) Defining and identifying communities in networks. PNAS 101:2658-2663

83. Newman MEJ (2004) Fast algorithm for detecting community structure in networks. Phys Rev E 69:066133

84. Sun S, Dong X, Fu Y, Tian W (2011) An iterative network partition algorithm for accurate identification of dense network modules. Nucleic Acids Res 
85. Glaab E, Baudot A, Krasnogor N, Valencia A (2010) Extending pathways and processes using molecular interaction networks to analyse cancer genome data. BMC Bioinform 11:597

86. Nitsch D et al (2011) PINTA: a web server for network-based gene prioritization from expression data. Nucleic Acids Res 39:W334

87. Jonsson PF, Bates PA (2006) Global topological features of cancer proteins in the human interactome. Bioinformatics 22:2291-2297

88. Liu CC et al (2006) Topology-based cancer classification and related pathway mining using microarray data. Nucleic Acids Res 34:4069-4080

89. Wang Q et al. (2011) A novel network-based method for measuring the functional relationship between gene sets. Bioinformatics doi:10.1093/bioinformatics/btr154

90. Alexeyenko A et al (2012) Network enrichment analysis: extension of gene-set enrichment analysis to gene networks. BMC Bioinform 13:226

91. Glaab E, Baudot A, Krasnogor N, Schneider R, Valencia A (2012) EnrichNet: networkbased gene set enrichment analysis. Bioinformatics 28:i451-i457

92. Lee H, Tu Z, Deng M, Sun F, Chen T (2006) Diffusion kernel-based logistic regression models for protein function prediction. OMICS. J Integr Biol 10:40-55

93. Ma X, Tarone AM, Li W (2008) Mapping genetically compensatory pathways from synthetic lethal interactions in yeast. PLoS ONE 3:e1922

94. Li S, Wu L, Zhang Z (2006) Constructing biological networks through combined literature mining and microarray analysis: a LMMA approach. Bioinformatics 22:2143-2150

95. Glass L, Kauffman SA (1973) The logical analysis of continuous, non-linear biochemical control networks. J Theor Biol 39:103-129

96. Fauré A, Naldi A, Chaouiya C, Thieffry D (2006) Dynamical analysis of a generic Boolean model for the control of the mammalian cell cycle. Bioinformatics (Oxford, England) 22, e124-131

97. Garg A, Mendoza L, Xenarios I, DeMicheli G (2007) Modeling of multiple valued gene regulatory networks. In: Conference proceedings: annual international conference of the ieee engineering in medicine and biology society. IEEE engineering in medicine and biology society. Conference 2007, 1398-1404

98. Mendoza L, Xenarios I (2006) A method for the generation of standardized qualitative dynamical systems of regulatory networks. Theor Biol Med Model 3:13

99. Willadsen K, Wiles J (2007) Robustness and state-space structure of Boolean gene regulatory models. J Theor Biol 249:749-765

100. Ribeiro AS, Kauffman SA (2007) Noisy attractors and ergodic sets in models of gene regulatory networks. J Theor Biol 247:743-755

101. Garg A, Mohanram K, Di Cara A, De Micheli G Xenarios I (2009) Modeling stochasticity and robustness in gene regulatory networks. Bioinformatics (Oxford, England) 25, i101-109

102. Thomas R (1973) Boolean formalization of genetic control circuits. J Theor Biol 42:563-585

103. Sánchez-Corrales Y-E, Alvarez-Buylla ER, Mendoza L (2010) The Arabidopsis thaliana flower organ specification gene regulatory network determines a robust differentiation process. J Theor Biol 264:971-983

104. Wittmann DM et al (2009) Transforming boolean models to continuous models: methodology and application to T-cell receptor signaling. BMC Syst Biol 3:98

105. Krumsiek J, Pölsterl S, Wittmann DM, Theis FJ (2010) Odefy-from discrete to continuous models. BMC Bioinf 11:233

106. Li S, Brazhnik P, Sobral B, Tyson JJ (2008) A quantitative study of the division cycle of Caulobacter crescentus stalked cells. PLoS Comput Biol 4:e9

107. Tyson JJ, Csikasz-Nagy A, Novak B (2002) The dynamics of cell cycle regulation. BioEssays: News Rev Mol, Cell Dev Biol 24:1095-1109

108. Jaeger J et al (2004) Dynamical analysis of regulatory interactions in the gap gene system of drosophila melanogaster. Genetics 167:1721-1737 
109. Chen $\mathrm{KC}$ et al (2004) Integrative analysis of cell cycle control in budding yeast. Mol Biol Cell 15:3841-3862

110. Locke JCW et al. (2005) Extension of a genetic network model by iterative experimentation and mathematical analysis. Mol Syst Biol 1, 2005.0013

111. Klipp E, Herwig R, Kowald A, Wierling C, Lehrach H (2005) Systems biology in practice: concepts, implementation and application. (Wiley-VCH)

112. Cao J, Qi X, Zhao H (2012) In: next generation microarray bioinformatics. Wang J, Tan AC, Tian T (eds) 802, 185-197 Humana Press

113. Teusink B, Walsh MC, Van Dam K, Westerhoff HV (1998) The danger of metabolic pathways with turbo design. Trends Biochem Sci 23:162-169

114. Jung YK, Kim TY, Park SJ, Lee SY (2010) Metabolic engineering of escherichia coli for the production of polylactic acid and its copolymers. Biotechnol Bioeng 105:161-171

115. Vogel G (2010) For more protein filet of cricket. Science 327:811

116. Hornberg JJ, Bruggeman FJ, Westerhoff HV, Lankelma J (2006) Cancer: a systems biology disease. Biosystems 83:81-90

117. Bakker BM, Westerhoff HV, Opperdoes FR, Michels PAM (2000) Metabolic control analysis of glycolysis in trypanosomes as an approach to improve selectivity and effectiveness of drugs. Mol Biochem Parasitol 106:1-10

118. Bakker BM et al. (2000) Compartmentation protects trypanosomes from the dangerous design of glycolysis. Proc Natl Acad Sci U S A 97, 2087-92

119. Alarcon $\mathrm{T}$ (2006) In mathematics, developmental biology and tumour growth: UIMPRSME Lluis A. Santaló Summer School, September 11-15, 2006, Universidad Internacional Menéndez Pelayo, Santander, Spain 45-74 (American mathematical soc, 2009)

120. Osborne JM et al (2010) A hybrid approach to multi-scale modelling of cancer. Phil Trans R Soc A 368:5013-5028

121. Fischer $\mathrm{S}$ et al. (2012) Modeling erythroblastic islands: using a hybrid model to assess the function of central macrophage. 92-106

122. Huang S, Ernberg I, Kauffman S (2009) Cancer attractors: a systems view of tumors from a gene network dynamics and developmental perspective. Semin Cell Dev Biol 20:869-876

123. Del Sol A, Balling R, Hood L, Galas D (2010) Diseases as network perturbations. Curr Opin Biotechnol 21:566-571

124. Alon U (2007) An introduction to systems biology: design principles of biological circuits. (Chapman \& Hall/CRC)

125. Materna SC, Nam J, Davidson EH (2010) High accuracy, high-resolution prevalence measurement for the majority of locally expressed regulatory genes in early sea urchin development. 177-184

126. Jurkowski W, Roomp K, Crespo I, Schneider JG, Del Sol A (2011) PPAR $\gamma$ population shift produces disease-related changes in molecular networks associated with metabolic syndrome. Cell Death Dis 2:e192

127. Crespo I, Roomp K, Jurkowski W, Kitano H, Del Sol A (2012) Gene regulatory network analysis supports inflammation as a key neurodegeneration process in prion disease. BMC Syst Biol 6:132

128. Huang S, Eichler G, Bar-Yam Y, Ingber DE (2005) Cell fates as high-dimensional attractor states of a complex gene regulatory network. Phys Rev Lett 94:128701

129. Maamar H, Raj A, Dubnau D (2007) Noise in gene expression determines cell fate in Bacillus subtilis. Science (New York) 317, 526-529

130. Gordon AJE et al (2009) Transcriptional infidelity promotes heritable phenotypic change in a bistable gene network. PLoS Biol 7:e44

131. Shiraishi T, Matsuyama S, Kitano H (2010) Large-scale analysis of network bistability for human cancers. PLoS Comput Biol 6:e1000851

132. Tafforeau L, Rabourdin-Combe C, Lotteau V (2012) In two hybrid technologies. Suter B, Wanker EE (eds) 812, Humana Press, pp 103-120 
133. Apsel B et al (2008) Targeted polypharmacology: discovery of dual inhibitors of tyrosine and phosphoinositide kinases. Nat Chem Biol 4:691-699

134. Achenbach J, Tiikkainen P, Franke L, Proschak E (2011) Computational tools for polypharmacology and repurposing. Future med chem 3:961-968

135. Rask-Andersen M, Almén MS, Schiöth HB (2011) Trends in the exploitation of novel drug targets. Nat Rev Drug Discov 10:579-590

136. Berger SI, Iyengar R (2011) Role of systems pharmacology in understanding drug adverse events. Wiley Interdisc Rev: Syst Biol Med 3:129-135

137. Gertsch J (2011) Botanical drugs, synergy, and network pharmacology: forth and back to intelligent mixtures. Planta Med 77:1086-1098

138. Arrell DK, Terzic A (2010) Network systems biology for drug discovery. Clin Pharmacol Ther 88:120-125

139. Berger SI, Iyengar R (2009) Network analyses in systems pharmacology. Bioinformatics 25:2466-2472

140. Zhao S, Li S (2010) Network-based relating pharmacological and genomic spaces for drug target identification. doi:10.1371/journal.pone.0011764

141. Azuaje FJ, Zhang L, Devaux Y, Wagner DR (2011) Drug-target network in myocardial infarction reveals multiple side effects of unrelated drugs. Scientific reports 1 